Araştırma Makalesi - Research Article

\title{
Kaldırma Düzeninde Kafes Uygulaması
}

Geliş / Received: 12/06/2019

Mehmet Cemil KALE ${ }^{1 *}$

Revize / Revised: 10/09/2019

Kabul / Accepted: 02/10/2019

$\ddot{\mathbf{O Z Z}}$

Filtre bankalarında altbant, kaldırma ve altbantın bir parçası olarak kafes düzenleri kullanılmaktadır. Bunların içinden kaldırma düzeni, işareti alt örneklemeyi daha önce yaparak aritmetik işlem sayısını yarı yarıya indirdiği için daha avantajlıdır. Ancak, kafes tekniği kaldırma düzeninde kullanılmamaktadır. Bu makalede anlatılan araştırmada, filtre bankaları için kaldırma düzeninde kafes uygulaması geliştirilmiştir. Mükemmel geri oluşturulma için tüm koşullar ele alınmış ve detaylıca açıklanmıştır. Geliştirilen düzen 4 test resmi üzerinde denenmiş ve geleneksel kafes düzenine göre daha iyi sonuçlar elde edilmiştir. 


\title{
Lattice Application on the Lifting Scheme
}

\begin{abstract}
In the filter banks, subband scheme, lifting scheme and as a part of the subband scheme, the lattice scheme are used. Among these, lifting scheme has the advantage of decreasing the arithmetic operations by half, because it downsamples the signal first. However, lattice is not used in the lifting scheme. In the research described in this article, lattice application for filter banks on the lifting scheme has been developed. Also, the constraints for the perfect reconstruction are given in detail. The performance of the new scheme is tested on 4 test images and better results are obtained compared to the traditional lattice scheme.
\end{abstract}

Keywords- Lattice Scheme, Lifting Scheme, Filter Banks 


\section{GİRIŞ}

İşaret işleme uygulamalarında en yaygın filtre bankası tekniklerinden birisi dörtlü ayna filtre (DAF) tekniğidir. Bu teknikte, analiz kısmında işaretler filtreleme ve alt örnekleme işlemleri sonrası iki ayrı parçaya ayrılır ve sentez kısmında bu parçalar filtreleme ve üst örnekleme işlemlerinden geçirilerek işaret yeniden oluşturulur. Mükemmel geri oluşturma, DAF bankaları alanının en önemli şartıdır. DAF bankaları, geleneksel altbant düzeni, kaldırma düzeni ve altbant düzeninin bir parçası olan kafes düzenleri gibi teknikleri içermektedir.

DAF tekniği, bir örtüşme engelleme metodu olarak Nussbaumer tarafindan ortaya atılmış ve çeşitli araştırmacılar tarafından geliştirilmiştir [1-5]. Bu araştırmaları, M kanallı filtre bankalarının genel teorisiyle ilgili çalışmalar takip etmiştir [6-14]. Filtre bankalarının yaygın kullanım alanlarından birisi de dikgen ve çift dikgen dalgacık dönüşüm teknikleridir.

Kaldırma düzeni etkin olarak çift dikgen dalgacık dönüşümlerinde kullanılmaktadır [15-23]. Öte yandan, geleneksel kafes yapısı, 2 kanallı DAF bankalarında uygulanmaktadır ve bir altbant filtre bankası uygulamasıdır [24-25]. Kafes yapısının avantajı sade işlemlerden oluşmasıdır. Geleneksel kafes yapısı altbant düzeninde uygulanırken, kaldırma düzeninde uygulanmamaktadır. Bu araştırmada yeni bir çalışma olarak kafes yapısı, kaldırma düzenine uyarlanmaktadır. $\mathrm{Bu}$ araştırmadan önce kaldırma düzeninde kafes yapısı uygulanmamıştır. $\mathrm{Bu}$ yeni düzende kafes yapısı kaldırma düzenine uyarlanırken, mükemmel geri oluşturma koşulları göz önüne alınacaktır. Ancak belirtilmelidir ki, bu makalede öne sürülen yeni yapı, bir dalgacık dönüşümü değil, bir filtre bankasıdır.

Makale, Bölüm 2' de geleneksel kafes yapısı anlatılarak devam edecektir. Geleneksel kafes yapısının ardından, ilk defa bu araştırmada tasarlanmış kaldırmalı kafes yapısı Bölüm 3' te tanıtılacaktır. Bölüm 4' te ise bu çalışmada geliştirilmiş olan kaldırmalı kafes düzeninin yapısı ve çalışma koşulları irdelenecektir. Son olarak da sonuç kısmında deneysel sonuçlar verilerek çalışmanın katkılarıtoparlanacaktır.

\section{GELENEKSEL KAFES YAPISI}

Geleneksel kafes yapısı dörtlü ayna filtreler için tasarlanmıştır [24-25]. Bu yapının analiz kısmı Şekil 1' de gösterilmektedir [24-25].

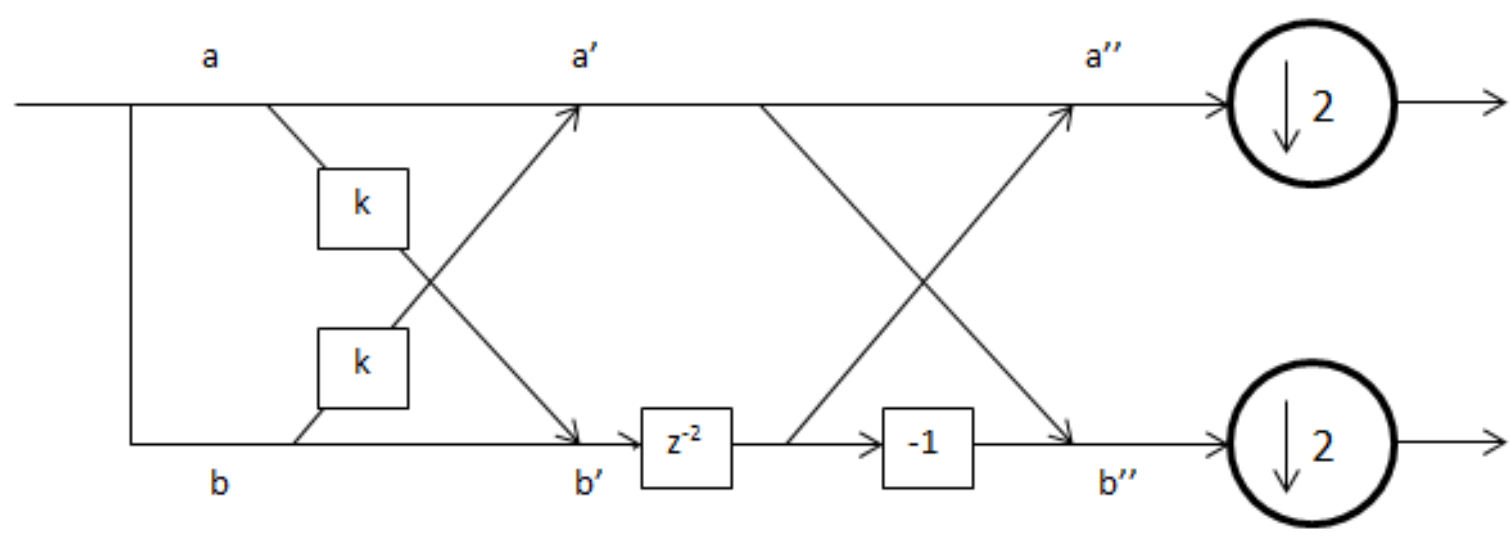

Şekil 1. Geleneksel kafes yapısının analiz kısmı

Yukarıdaki Şekil 1' de, a, a', a", b, b' ve b" düğüm noktalarını belirlemek için kullanılan değişkenlerdir. Öte yandan $k$ ise bir skalar çarpım elemanıdır. Şekilde görüldüğü üzere, işaret önce [a,b] ve [a",b"] düğümleri arasında bir filtreleme işleminden geçerken, [a", b"] düğümü sonrasında filtrelenmiş işaretlerin alt örneklemesi yapılmaktadır. Kafes yapısının avantajı, şekilde görüldüğü üzere, basit skalar 
çarpımlardan $(k$ ya da -1$)$ ve geciktirmelerden $\left(z^{-2}\right)$ oluşmasıdır. Bu nedenle kafes yapısının gerçekleştirmesi son derece kolaydır.

Geleneksel kafes yapısının sentez kısmı ise Şekil 2’ de gösterilmektedir [23-24].

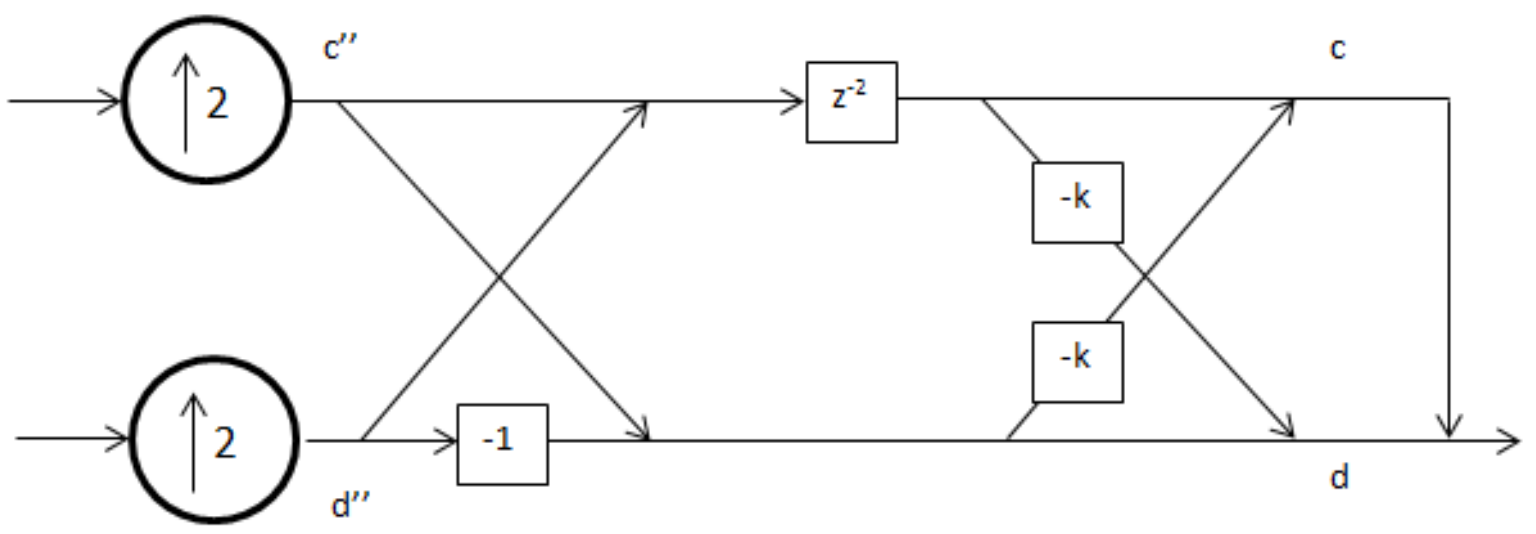

Şekil 2. Geleneksel kafes yapısının sentez kısmı

Yine, bu şekilde c, c', d ve d" düğüm noktaları iken k skalar çarpım elemanıdır. İşaretlere [c', d'] düğümünden hemen önce üst örnekleme yapılmasının ardından, işaretler [c', d' '] ve [c, d] düğümleri arasındaki filtrelerden geçirilerek toplanmakta ve mükemmel geri oluşum tamamlanmaktadır [24-25].

Şekil 1 ve 2 göstermektedir ki, kafes yapısı aslında altbant filtreleri ile işaretin ayrıldı ̆̆ bir altbant uygulamasıdır. Bu araştırma, kafes yapısını, filtrelemelerin önce yapıldığı bir altbant modeli olarak değil de, alt örneklemenin önce yapıldığı bir kaldırma modeli olarak tasarlamayı amaçlamaktadır.

\section{KALDIRMALI KAFES YAPISI}

Kaldırma düzeni bir dalgacık tasarım tekniği olmasının yanı sıra bir dalgacık dönüşüm tekniğidir. Kaldırma düzeninde bir işaret, iki parçaya ayrılarak (işaret ve aynı işaretin 1 birim geciktirilmiş halinin alt örneklemeleri yapılarak) filtreleme işlemlerinden geçirilir. Bu düzende işlemler genelde basit kaydırma ve toplama işlemleridir. Kaldırma düzeninin tercih edilmesinin ana nedeni, alt örneklemenin önce yapılması sebebiyle aritmetik işlemlerin yarı yarıya azalmasıdır [15-17].

Kaldırmalı kafes yapısının analiz kısmı Şekil 3' te gösterilmektedir. Bu modelde, alt örneklemeler önce yapılarak, işaret bir fermuar gibi ikiye ayrılmaktadır. 


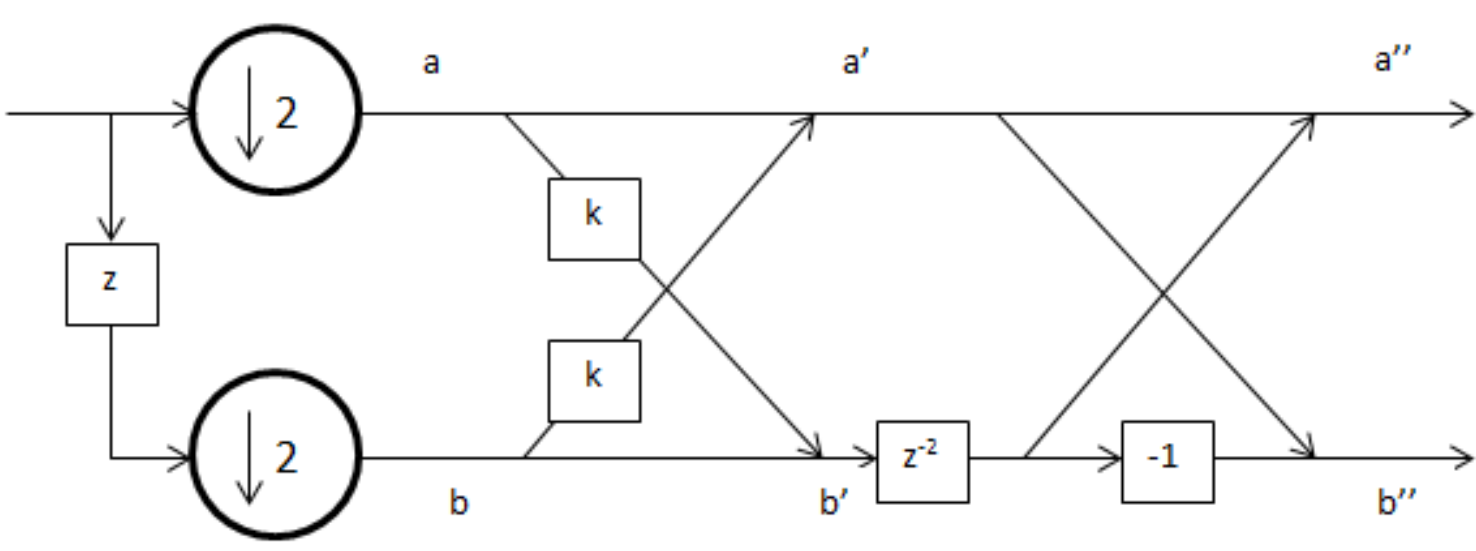

Şekil 3. Kaldırmalı kafes yapısının analiz kısmı

Şekil 3' e dikkatli bakıldığında, filtreleme işlemlerinden önce, işarete, yukarıdaki branşta alt örnekleme uygulandığı ve işaretin çift elemanlarının $(n=0,2,4, \ldots)$ alındığı görülecektir (Düğüm a). Yine aynı şekilde, bir kaydırmanın $(z)$ ardından,işarete alt branşta alt örnekleme uygulanmış ve bu sefer de işaretin tek elemanları $(n=1$, $3,5, \ldots$ ) alınmıştır (Düğüm b). Artık işlenen işaretlerçift ve tek olarak ayrılmıştır. İşaretlerin boyutu yarı yarıya düşmüştür ve bu da aritmetik işlemleri yarı yarıya düşürecektir [15-17].

Eşitlikler 1 ve 2 yardımıyla analiz kısmının çok fazlı matrisi bulunabilir.

$$
\begin{aligned}
& {\left[\begin{array}{l}
a^{\prime} \\
b^{\prime}
\end{array}\right]=\left[\begin{array}{ll}
1 & k \\
k & 1
\end{array}\right]\left[\begin{array}{l}
a \\
b
\end{array}\right]} \\
& {\left[\begin{array}{l}
a^{\prime \prime} \\
b^{\prime \prime}
\end{array}\right]=\left[\begin{array}{cc}
1 & z^{-2} \\
1 & -z^{-2}
\end{array}\right]\left[\begin{array}{l}
a \prime \\
b^{\prime}
\end{array}\right]}
\end{aligned}
$$

Eşitlikler 1 ve 2 birleştirilerek kaldırmalı kafes yapısının analiz safhası çok fazlı matrisi aşağıda Eşitlik 3' te gösterilmektedir.

$$
\left[\begin{array}{l}
a \prime \prime \\
b^{\prime \prime}
\end{array}\right]=\left[\begin{array}{ll}
1+k z^{-2} & k+z^{-2} \\
1-k z^{-2} & k-z^{-2}
\end{array}\right]\left[\begin{array}{l}
a \\
b
\end{array}\right]
$$

Mükemmel geri yapılandırma için işler bir sentez oluşturmak gerekmektedir. Sentez kısmının çok fazlı matrisi, analiz safhasının çok fazlı matrisinin tersidir [15-17]. Sentezçok fazlı matrisinin, analiz çok fazlı matrisinin tersi olması, mükemmel geri yapılandırma için yeterli bir koşuldur [15-17]. Bu nedenle, sentez elde edebilmek için analiz safhasının çok fazlı matrisinin tersinin alınabilmesi gerekmektedir. Çok fazlı analiz matrisinin tersinin alınabilmesi için, bu matrisin determinantının 0' dan farklı olması gerekmektedir. Bu matrisin determinantı Eşitlik 4' te gösterilmektedir.

$$
\operatorname{det}\left(\left[\begin{array}{ll}
1+k z^{-2} & k+z^{-2} \\
1-k z^{-2} & k-z^{-2}
\end{array}\right]\right)=2\left(k^{2}-1\right) z^{-2}
$$

Yukarıdaki eşitliklerden yola çıkarak sentez safhasının yapısı Şekil 4' te sergilenmekte ve sentez safhasının çok fazlı matrisi ise Eşitlik 5' te gösterilmektedir. 


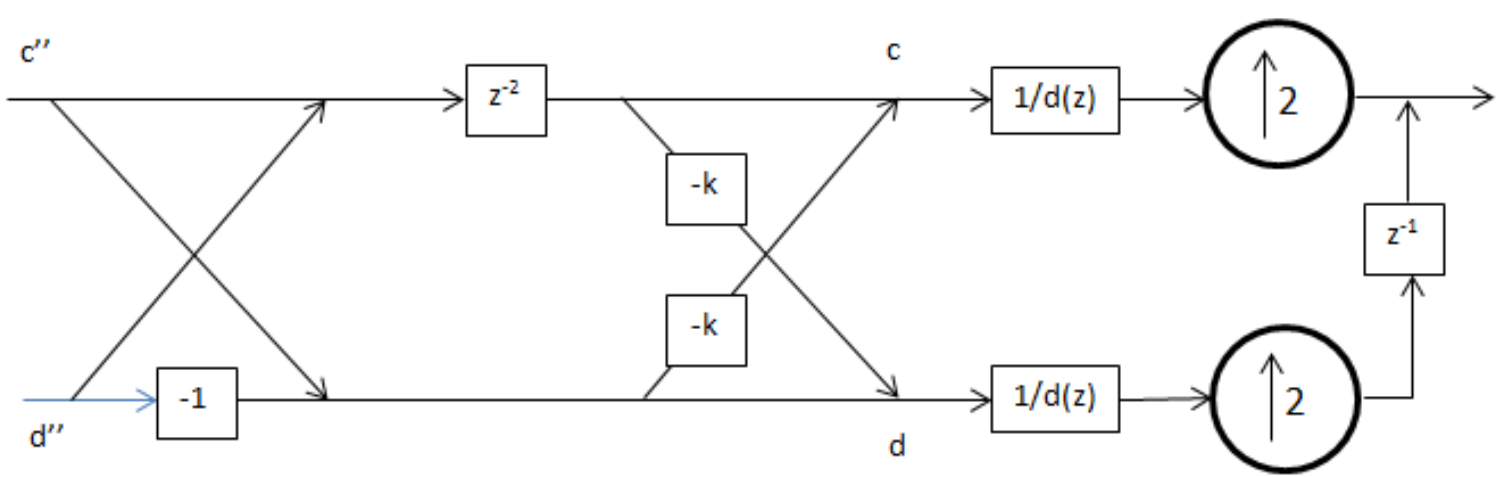

Şekil 4. Kaldırmalı kafes yapısının sentez kısmı

$$
\left[\begin{array}{l}
c \\
d
\end{array}\right]=\frac{1}{2\left(k^{2}-1\right) z^{-2}}\left[\begin{array}{cc}
k-z^{-2} & -k-z^{-2} \\
-1+k z^{-2} & 1+k z^{-2}
\end{array}\right]\left[\begin{array}{c}
c^{\prime \prime} \\
d \prime \prime
\end{array}\right]
$$

Şekil 4' te $d(z)$, analiz çok fazlı matrisinin determinantından gelen etkidir. Burada, $d(z)=2\left(k^{2}-1\right) z^{-2}$ olarak tanımlanmaktadır. Her koşulda $1 / d(z)$ filtresinin kutupları $|z|=1$ dairesinin içerisinde kalacağından bu sistem kararlıdır.

\section{KAFES YAPISI TASARIM KOŞULLARI}

Kafes yapısının genel bir çerçevede tasarım kuralları iyi belirlenmelidir. Her zaman $z^{-2}$, lik bir geciktirici kullanılmayabilir. Şekiller 1-4' te bulunan $z^{-2}$ geciktiricisi yerine bir sonlu dürtü tepkisi filtresi olarak $N(z)$ kullanılabilir. Şekil 3-4' teki $z^{-2}, N(z)$ ile değiştirildiğinde ve Eşitlikler 6 ve 7 kullanıldığında,

$$
\begin{aligned}
& {\left[\begin{array}{l}
a^{\prime} \\
b^{\prime}
\end{array}\right]=\left[\begin{array}{ll}
1 & k \\
k & 1
\end{array}\right]\left[\begin{array}{l}
a \\
b
\end{array}\right]} \\
& {\left[\begin{array}{l}
a^{\prime \prime} \\
b^{\prime \prime}
\end{array}\right]=\left[\begin{array}{cc}
1 & N(z) \\
1 & -N(z)
\end{array}\right]\left[\begin{array}{l}
a \prime \\
b^{\prime}
\end{array}\right]}
\end{aligned}
$$

tasarlanan bu genel kafes yapısının çok fazlı analiz matrisi aşağıda, Eşitlik 8' de gösterilmektedir.

$$
\left[\begin{array}{l}
a \prime^{\prime \prime} \\
b_{\prime \prime}^{\prime \prime}
\end{array}\right]=\left[\begin{array}{ll}
1+k N(z) & k+N(z) \\
1-k N(z) & k-N(z)
\end{array}\right]\left[\begin{array}{l}
a \\
b
\end{array}\right]
$$

Mükemmel yeniden yapılandırma için, yani analiz safhası çok fazlı matrisin tersinin alınabilmesi için, analiz çok fazlı matrisinin determinantının 0’ dan farklı olması gerekmektedir. Bu determinant aşağıda Eşitlik 9' da gösterilmektedir.

$$
\operatorname{det}\left(\left[\begin{array}{ll}
1+k N(z) & k+N(z) \\
1-k N(z) & k-N(z)
\end{array}\right]\right)=2\left(k^{2}-1\right) N(z)
$$

Analiz çok fazlı matrisinin tersi olan sentez kısmının çok fazlı matrisi ise aşağıda, Eşitlik 10’ da gösterilmektedir.

$$
\left[\begin{array}{l}
c \\
d
\end{array}\right]=\frac{1}{2\left(k^{2}-1\right) N(z)}\left[\begin{array}{cc}
k-N(z) & -k-N(z) \\
-1+k N(z) & 1+k N(z)
\end{array}\right]\left[\begin{array}{c}
c^{\prime \prime} \\
d^{\prime \prime}
\end{array}\right]
$$

$\mathrm{Bu}$ sentez modelinin gerçekleştirilebilmesi, başka bir dille sistemin kararlı olabilmesi için $N(z)$ filtresinin sıfırlarının $|z|=1$ dairesinin içerisinde kalması gerekmektedir. Sistemin kararsız olması durumunda, işaret yeniden yapılandırma aşamasında istenmeyen noktalara gidebileceğinden (örneğin işaretin sonsuza doğru 
yükselmesi gibi), sistemin kararlı olması yüksek önem gerektirmektedir. Özetle kararsız sistemlerde mükemmel yeniden yapılanma sağlanamaz.

\section{V.SONUÇ}

Filtre bankalarında istenen, alt branşta yani yüksek bantta çıkan işaretin varyansının düşük olmasıdır. Çeşitli test resimleri için yüksek bant (alt branş) ve düşük bant (üst branş) varyansları Tablo 1 ve 2' de gösterilmektedir. Bu deneylerde, filtre bankası, resimlerin satırlarına uygulanmıştır.

Tablo 1. Düşük bant işaretlerin varyansları

\begin{tabular}{lll}
\hline & $\begin{array}{c}\text { Kafes Düzeni } \\
(\mathbf{k = 1})\end{array}$ & $\begin{array}{c}\text { Kaldırmalı } \\
\text { Kafes Düzeni } \\
(\mathbf{k = 0 . 2})\end{array}$ \\
\hline Barbara & 42336 & 15387 \\
Biberler & 45700 & 16027 \\
Elaine & 34121 & 11979 \\
Lenna & 43004 & 14995
\end{tabular}

Tablo 2. Yüksek bant işaretlerin varyansları

\begin{tabular}{lll}
\hline & $\begin{array}{c}\text { Kafes Düzeni } \\
(\mathbf{k = 1})\end{array}$ & $\begin{array}{c}\text { Kaldırmalı } \\
\text { Kafes Düzeni } \\
(\mathbf{k}=\mathbf{0 . 2})\end{array}$ \\
\hline Barbara & 2473 & 1421 \\
Biberler & 1188 & 977 \\
Elaine & 831 & 853 \\
Lenna & 1559 & 1276
\end{tabular}

Tablo 2’ de görüldüğü üzere $\mathrm{k}$ sabiti \%80 azaltılarak yüksek bant parçada daha iyi sonuçlar (daha düşük varyans) elde edilmektedir. Bu modelde $N(z)=z^{-2}$ kullanılmıştır. Kaldırma yapısının bir diğer önemli avantajı ise, bu düzende filtre bankası işleminden önce, işaret bir fermuar gibi iki ayrı parçaya bölünmektedir [15-17]. Bunun sonucu olarak da işlem adedi yarı yarıya azaltılmaktadır. Bu nedenle bu metodun geleneksel kafes yapısından daha hızlı bir teknik olduğu da belirtilebilir.

$\mathrm{Bu}$ çalışmada, altbant düzeninde kullanılan kafes yapısının, aritmetik işlemleri yarı yarıya indiren kaldırma yapısında kullanılması anlatılmıştır. Kaldırma düzeninde, çok fazlı analiz matrisiyle dönüşüm yapmak öne çıkmaktadır. Bu düzende, analiz çok fazlı matrisinin tersi ise sentez safhasının çok fazlı matrisidir. Bu nedenle, matrislerin tersinin alınabilmesi gerekmektedir. Matrislerin tersinin alınabilmesi de tüm sorunları çözmemektedir. Bu matrislerin determinantları, matrislerin tersinde payda etkisi göstermektedir. Bu yüzden analiz çok fazlı matrisinin determinantının sıfırlarının (veya sentez safhası çok fazlı matrisinin kutuplarının) $|z|=1$ dairesinin içerisinde kalması ve sistemin daima kararlı olması gerekmektedir.

\section{KAYNAKLAR}

[1] Nussbaumer, H. J. (1981). Pseudo QMF filter bank., IBM Tech. Disclosure Bulletin., 24, 3081-3087.

[2] Rothweiler, J. H. (1983). Polyphase Quadrature Filters, a New Subband Coding Technique. Proceedings of the IEEE International Conference on ASSP, 1983, Boston MA, 1980-1983.

[3] Chu, P.L. (1985). Quadrature Mirror Filter Design for an Arbitrary Number of Equal Bandwidth Channels. IEEE Transactions on Acoustics, Speech and Signal Processing., vol. ASSP-33, 203-218. 
[4] Masson, J. \& Picel, Z. (1985). Flexible Design of Computationally Efficient Nearly Perfect QMF Filter Banks.Proceedings of the IEEE International Conference on ASSP, 1985, Tampa FL, 14.7.1-14.7.4.

[5] Cox, R. V. (1986). The Design of Uniformly and Nonuniformly Spaced Pseudo QMF. IEEE Transactions on Acoustics, Speech and Signal Processing, vol. ASSP-34, 1090-1096.

[6] Ramstadt, T. A. (1984). Analysis/Synthesis Filter Banks with Critical Sampling. International Conference on Digital Signal Processing. 1984. Florence.

[7] Smith, M. J. T. \& Barnwell, III, T. P. (1985). A Unifying Framework for Analysis/Synthesis Systems Based on Maximally Decimated Filter Banks, Proceedings of the IEEE International Conference on ASSP, Tampa FL, 521-524

[8] Vetterli, M. (1985). Splitting a Signal into Subsampled Channels Allowing Perfect Reconstruction, Proc. IASTED Conf. Appl. Signal Proc., 1985, Paris.

[9] Princen, J. P. \& Bradley, A. P. (1986).Analysis/Synthesis Filter Bank Design Based on Time Domain Aliasing Cancellation, IEEE Transactions on Acoustics, Speech and Signal Processing, vol. ASSP-34, 1153-1161.

[10] Wackershruther, G. (1986). Some New Aspects of Filters for Filter Banks. IEEE Transactions on Acoustics, Speech and Signal Processing, vol. ASSP-34, 1182-1200.

[11] Vaidyanathan P. P. (1987). Theory and Design of M-Channel Maximally Decimated Quadrature Mirror with Arbitrary M, Having Perfect Reconstruction Property. IEEE Transactions on Acoustics, Speech and Signal Processing, Vol. ASSP-35, 476-492.

[12] Vaidyanathan P. P. (1987). Quadrature Mirror Filter Banks, M-Band Extensions and Perfect Reconstruction Techniques. IEEE ASSP magazine, vol 4, 4-20.

[13] Nguyen T. Q. \&Vaidyanathan P. P. (1988).Two Channel Perfect Reconstruction FIR QMF Structures which Yield Linear Phase FIR Analysis and Synthesis Filters. IEEE Transactions on Acoustics, Speech and Signal Processing, vol. ASSP-38, 676-690.

[14] Viscito \& Allebach (1988). The Desing of Tree Structured M-channel filter banks using Perfect Reconstruction Filter Blocks. Proceedings of the IEEE International Conference on ASSP, New York, $1475-1478$.

[15] Sweldens, W. (1996) The Lifting Scheme: A Custom-Design Construction of Biorthogonal Wavelets. Applied and Computational Harmonic Analysis, 3(2), 186-200.

[16] Sweldens, W. (1997). The lifting scheme: A construction of second generation wavelets. SIAM J. Math. Anal., 29(2), 511-546.

[17] Daubechies I. \& Sweldens W. (1998). Factoring wavelet transforms into lifting steps, Journal of Fourier Analysis and Applications, 4(3), 247-269.

[18] Gerek, Ö. N. \& Cetin, A. E. (2000). Adaptive polyphase subband decomposition structures for image compression. IEEE Transactions on Image Processing, 9(10), 1649-1660.

[19] Gerek, Ö. N. \& Cetin, A. E. (2006). A 2-D orientation- adaptive prediction filter in lifting structures for image coding. IEEE Transactions on Image Processing, 15(1), 106-111.

[20] Habiboglu Y. H., Kose K., \& Cetin A. E. (2011) Fractional wavelet transform using an unbalanced lifting structure, Proc. SPIE 8058, Indepen- dent Component Analyses, Wavelets, Neural Networks, Biosystems, and Nanoengineering IX, 805805. 
[21] Keskin F. \& A. E. Cetin A. E. (2011). Directionally selective fractional wavelet transform using a 2-D nonseparable unbalanced lifting structure. In: E. Salerno, A. E. Cetin, O. Salvetti (eds) Computational Intelligence for Multimedia Understanding. MUSCLE 2011. Lecture Notes in Computer Science, vol 7252. Springer, Berlin, Heidelberg

[22] Kale, M. C., Atac, G., \& Gerek, Ö. N. (2016), A Biorthogonal Wavelet Design Technique using KarhunenLoeve Transform Approximation, Digital Signal Processing, 51(4), 202-222.

[23] Kale M. C. (2016). A general biorthogonal wavelet based on Karhunen-Loeve transform approximation, Signal, Image and Video Processing, 10(4), 791-794.

[24] Tran T. D., de Queiroz R. L. \& Nguyen T. Q. (2000). Linear-phase perfect reconstruction filter bank: lattice structure, design, and application in image coding, IEEE Transactions on Signal Processing, 48(1), 133147.

[25] Vaidyanathan P. P. (1993). Multirate Systems and Filter Banks, Prentice Hall. 